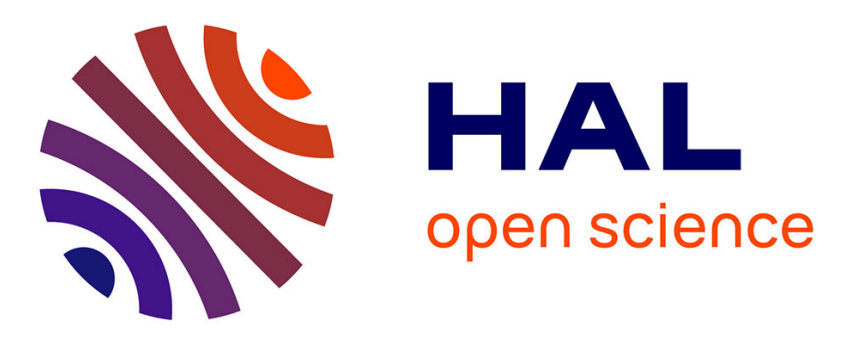

\title{
Neural mechanisms associated with treatment decision making: An fMRI study
}

\author{
Malek Abidi, Jared Bruce, Alain Le Blanche, Amanda Bruce, David P \\ Jarmolowicz, Antonia Csillik, N. Jade Jade Thai, Seung-Lark Lim, Olivier \\ Heinzlef, Giovanni de Marco
}

\section{To cite this version:}

Malek Abidi, Jared Bruce, Alain Le Blanche, Amanda Bruce, David P Jarmolowicz, et al.. Neural mechanisms associated with treatment decision making: An fMRI study. Behavioural Brain Research, 2018, 349, pp.54-62. 10.1016/j.bbr.2018.04.034 . hal-02310462

\section{HAL Id: hal-02310462 \\ https://hal.parisnanterre.fr/hal-02310462}

Submitted on 10 Oct 2019

HAL is a multi-disciplinary open access archive for the deposit and dissemination of scientific research documents, whether they are published or not. The documents may come from teaching and research institutions in France or abroad, or from public or private research centers.
L'archive ouverte pluridisciplinaire HAL, est destinée au dépôt et à la diffusion de documents scientifiques de niveau recherche, publiés ou non, émanant des établissements d'enseignement et de recherche français ou étrangers, des laboratoires publics ou privés. 


\title{
Neural mechanisms associated with treatment decision making: An fMRI study
}

\author{
Malek Abidi ${ }^{\mathrm{a}, *}$, Jared Bruce ${ }^{\mathrm{b}, \mathrm{c}}$, Alain Le Blanche ${ }^{\mathrm{a}, \mathrm{d}}$, Amanda Bruce $^{\mathrm{e}}$, David P. Jarmolowicz \\ Antonia Csillik ${ }^{g}$, N. Jade Thai ${ }^{h}$, Seung-Lark Lim ${ }^{b}$, Olivier Heinzlef ${ }^{i}$, Giovanni de Marco ${ }^{\mathrm{a}}$ \\ ${ }^{\mathrm{a}}$ Laboratoire CeRSM (EA-2931), UPL, Université Paris Nanterre, F92000, Nanterre, France \\ ${ }^{\mathrm{b}}$ Department of Psychology, University of Missouri-Kansas City, Kansas City, USA \\ ${ }^{\mathrm{c}}$ Department of Biomedical and Health Informatics, Unviersity of Missouri - Kansas City \\ ${ }^{\mathrm{d}}$ Hôpital René-Dubos de Pontoise and Université de Versailles-Saint-Quentin, Simone Veil UFR des Sciences de la Santé, France \\ e Department of Pediatrics, Center for Children's Healthy Lifestyles \& Nutrition, University of Kansas Medical Center, Kansas City, USA \\ ${ }^{\mathrm{f}}$ Department of Applied Behavioral Science, University of Kansas, Lawrence, USA \\ ${ }^{\mathrm{E}}$ EA 4430, Clinique Psychanalyse et Développement (CLIPSYD), Paradigme empirique et Thérapies cognitivo-comportementales, Université Paris-Nanterre, 200 avenue de \\ la République, 92000, Nanterre, France \\ ${ }^{\mathrm{h}}$ Clinical Research \& Imaging Centre (CRIC Bristol), Bristol Medical School, University of Bristol, Bristol, UK \\ ${ }^{\mathrm{i}}$ CHI de Poissy St-Germain, F78300, France
}

A R T I C L E I N F O

Keywords:

Behavioral economic model

Treatment decision making

fMRI treatment decision probability

discounting

$\%$ BOLD signal change

Psychophysiological interaction

\begin{abstract}
A B S T R A C T
Great progress has been made in understanding how people make financial decisions. However, there is little research on how people make health and treatment choices. Our study aimed to examine how participants weigh benefits (reduction in disease progression) and probability of risk (medications' side effects) when making hypothetical treatment decisions, and to identify the neural networks implicated in this process.

Fourteen healthy participants were recruited to perform a treatment decision probability discounting task using MRI. Behavioral responses and skin conductance responses (SCRs) were measured. A whole brain analysis were performed to compare activity changes between "mild" and "severe" medications' side effects conditions. Then, orbitofrontal cortex (OFC), ventral striatum (VS), amygdala and insula were chosen for effective connectivity analysis.

Behavioral data showed that participants are more likely to refuse medication when side effects are high and efficacy is low. SCRs values were significantly higher when people made medication decisions in the severe compared to mild condition. Functionally, OFC and VS were activated in the mild condition and were associated with increased likehood of choosing to take medication (higher area under the curve "AUC" side effects/efficacy). These regions also demonstrated an increased effective connectivity when participants valued treatment benefits. By contrast, the OFC, insula and amygdala were activated in the severe condition and were associated with and increased likelihood to refuse treatment. These regions showed enhanced effective connectivity when participants were confronted with increased side effects severity. This is the first study to examine the behavioral and neural bases of medical decision making.
\end{abstract}

\section{Introduction}

Important decisions are often made that rely on probabilistic comparisons of uncertain costs and benefits. Great progress has been made in understanding how people make financial decisions [1-4]. Yet, there is relatively little research on how people make health and treatment choices [5,6]. Behavioral economics offers a methodology and perspective for quantitatively studying and understanding health decisions [7] using economic principles such us "probability discounting". For example, in recent studies, patients with multiple sclerosis (MS) indicated their likelihood of taking medications that had specified efficacies (i.e., benefits) and risks of side effects (i.e., risks) [8,9]. Patients indicated systematically lower likelihoods of taking medications as the efficacy declined and the risks of side effects increased. These treatment decision patterns were well-described by probability discounting models that are often used to describe choices regarding money [10,11] and other commodities [10-13]. Moreover, these choice patterns were significant predictors of patients' adherence status [8]. 
Understanding how patients make treatment decisions has substantial public health implications. As many as $50 \%$ of patients with chronic disease decline or prematurely discontinue prescribed treatment regimens even with highly efficacious medications. As a result, the World Health Organization concluded that improving treatment adherence would improve worldwide health more than most pharmacologic advances [14]. Health researchers frequently use bio-psychosocial theoretical models to better understand human behavior. Despite this, most adherence and treatment decision models rely exclusively on psychological or social constructs and very little is known about the neural mechanisms of treatment choice and adherence. Using a Treatment Decision Making fMRI Paradigm and existing literature that uses fMRI to examine brain mechanisms of risk and reward, the current study tested a neural model of hypothetical treatment choice in healthy adult participants. Gaining this initial understanding of the neural mechanisms of treatment choice may provide insights that can be applied to improve treatment adherence in chronic disease.

Decision neuroscience can be used to help understand how different brain regions work together to evaluate and act upon environmental stimuli. Many studies implicate reward representation $[15,16]$ and risk processing [1,2] brain networks, and have investigated the role of emotion [17-19] when people make monetary decisions. Moreover, discounting studies have implicated consistent neural circuitry for monetary and non-monetary rewards [20,21]. While several different brain regions have been shown to be involved in risk and reward, to the best of our knowledge no study has examined the roles of risk, reward and emotions in medical treatment decisions. In particular, functional connectivity between these brain regions has not been examined.

Adapting a behavioral economic model from our previous research $[8,9,22]$, the present study aimed to identify how people weigh the probability of benefits (i.e., efficacy) and risks (i.e., side effects) when deciding whether to take an hypothetical medication, and to examine the neural and affective mechanisms implicated in such treatment decisions.

We hypothesized that: 1) participants would be more likely to discount treatment benefits when confronted with an increased risk of side effects. 2) Because emotions play a crucial role in decision making [17-19], we predicted increased skin conductance responses (SCRs) when participants were confronted with decisions involving severe medication side effects compared to mild medication side effects. 3) Because the OFC and the VS are implicated in the representation of reward anticipation and processing $[15,16]$, we anticipated increased activity in these areas among participants who differentially value treatment benefits. Moreover, because animal and human studies provide evidence that adaptive decision-making and related psychological processes (e.g., reward valuation, anticipation, and motivation) are mediated through direct interactions between two key nodes of a posited "reward circuit", VS and OFC [23,24], we hypothesized increased connectivity between OFC-VS when people were making decisions involving mild medication side effects compared to severe medication side effects. 4) Finally, OFC, insula and amygdala have reciprocal anatomical connections, and are implicated in risk based decisionmaking. Consequently, we predicted increased activity in these regions when participants were confronted with more severe side effect profiles and an increased effective connectivity between OFC-insula and OFCamygdala in the severe medication side effects condition when compared to mild medication side effect condition.

\section{Materials and methods}

\subsection{Participants}

Fourteen healthy volunteers, (7 males and 7 females), from 18 to 29 years (average age $=23.50$; standard deviation $=3.75$ ), with master's degrees, right-handed, free of neurological disorders, not taking medications known to alter brain activity, and with no history of drug or alcohol abuse, participated in the study. Participants gave their informed, written consent and performed a hypothetical treatment decision making task during fMRI.

\section{2. fMRI paradigm}

The fMRI paradigm was adapted from a probability discounting task we have used in our previous research on patient treatment choice and adherence $[8,9,22]$. We employed an event-related decision making paradigm to evaluate treatment decision making in healthy adult participants. The instructions were clearly explained before starting the MRI paradigm and participants were asked to imagine they had multiple sclerosis (MS). We chose MS because it is a relatively common, chronic disease affecting adults that requires participants to take medications that have varying risk and reward profiles. We described the disease to the participants, how it progresses, as well as the impact of the disease on health and overall quality of life. We also explained that treatments reduce the disease activity, but may also have unwanted side effects. Side effect severities were then described to participants using definitions derived from adverse event reporting guidelines. Medication efficacies were described as the typical percent reduction in disease activity. Our reliance on a hypothetical choice paradigm rests upon previous work showing that hypothetical choices can provide a preliminary understanding of the neural underpinnings of how people weigh risks and benefits when making real-world treatment decisions. Prior studies provide evidence for the validity and the efficacy of similar hypothetical procedures [25-28].

The functional MRI paradigm presented a series of scenarios asking participants if they would take medications with a given probability of side effects $(1 \%, 5 \%, 16 \%, 38 \%, 64 \%, 84 \%, 95 \%, 99.9 \%)$ and reported medication efficacies $(1 \%, 5 \%, 16 \%, 38 \%, 64 \%, 84 \%, 95 \%, 99.9 \%)$. In total, participants were presented with $64(8 \times 8)$ choice scenarios for each of possible mild, moderate and severe side effects $(3 \times 64=192$ total choices). The scenarios were randomly intermixed over the task. We chose this broad range of values to be consistent with the real-world risk/benefit ratios of treatment and to ensure that participants would demonstrate sufficient variability in their responses for the proposed MRI analyses.

Two sessions of 96 choices were presented. Each session lasted about $15 \mathrm{~min}$. We divided the paradigm into two counterbalanced sessions in order to reduce any effects of fatigue on brain activation. We also included this break because we hope to further this research in clinical populations who may have difficulty engaging in experiments for sustained periods of time. There were no response time constraints. We chose not to include response constraints so that participants could fully analyze each risk/benefit scenario before making a treatment decision. When participants responded (made a choice), they proceeded directly to the next scenario. There was no jitter introduced in the experimental design. However, a sufficient degree of variability within subject was recorded $($ Mean $=5.60$, Range $=4.18-7.98$; Mean SD $=3.15$, Range $=\mathbf{2 . 0 - 3 . 8}$ )

Participants were asked to respond as rationally as possible (without any time constraint) to the following question: "would you take this medication?". Participants were presented with a binary choice (yes/ no) indicating whether they would take medication with each risk/ benefit ratio by using a 2 button response pad (Fig. 1).

\subsection{Behavioral responses and analysis}

Each participant's behavioral responses were aggregated for analysis in two ways: 1) Choices at all eight medication efficacies were collapsed at each side effect probability to examine effects of side effect probability (x-axis) on choices to take medications (i.e., number of "yes" responses / the eight medication efficacies; y-axis), and 2) Choices at each of the eight side effect probabilities were collapsed at each efficacy to examine effects of mediation efficacy (x-axis) on 


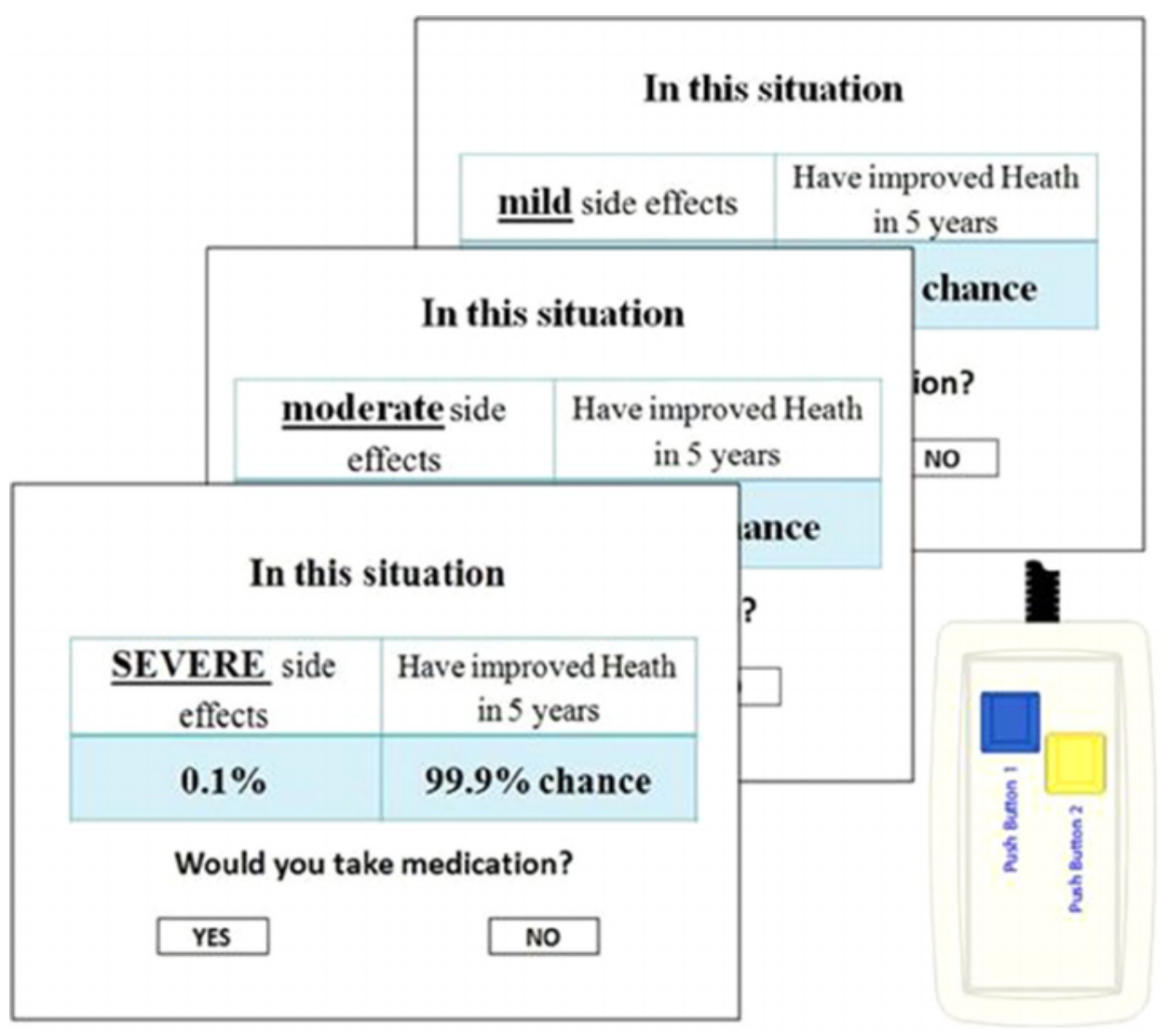

Fig. 1. Treatment decision making fMRI paradigm. A series of scenarios were presented asking about a participant's likelihood of taking medications when the probability of side effects and reported medication efficacies varied. Participants where then presented with a binary choice (yes/no) indicating whether they would take medication with each risk/ benefit ratio by using a 2 button response pad.

choices to take medications (i.e., number of "yes" responses / the eight side effect probabilities; $y$-axis).

Once aggregated, area under the curve (AUC) was calculated showing the main effects of side effect probability and efficacy at each of our three side effect severities. Area under the curve is frequently used $[29,30]$ to quantify discounting data because it provide a straight forward measure of discounting that is not linked to any theoretical framework [31]. Moreover, AUC provides a single number representing the entire discounting pattern, allowing analysis of relations between these patterns of behavioral economic decision making and neural activation. The obtained AUC values represent a proportion on a range between 0.0 and 1.0 where larger AUC values are indicative of slower or no discounting and lower AUC values mean greater levels of discounting.

Area under the curve was calculated using the trapezoid method [31] using the formula:

$\mathrm{AUC}=\sum\left(\mathrm{x}_{2}^{-} \mathrm{x}_{1}\right)\left[\frac{\mathrm{y}_{1}+\mathrm{y}_{2}}{2}\right]$

where $\mathrm{x}_{2}$ is the larger $\mathrm{x}$-axis value for each trapezoid, and $\mathrm{x}_{1}$ is the smaller $x$-axis value for each trapezoid and $y_{1}$ and $y_{2}$ are the $y$ values that correspond to those $\mathrm{x}$ coordinates. When calculating AUCs for side effects (at each side effect severity), the side effect probabilities were used for the $\mathrm{x}$ axis (with the percent yes corresponding to that probability being the corresponding y value), whereas probability of efficacy was used as the $\mathrm{x}$ axis for AUCs describing efficacy (at each side effect severity). Because the AUC values are calculated on a within subject basis, and are normally distributed, 2 (efficacy vs. side effect) x 3 side effect severity), a repeated measures ANOVA was used to determine main effects of side effect severity and side effect vs. efficacy.

\subsection{Skin conductance responses measurements}

Skin conductance responses (SCRs) were recorded continuously and simultaneously during the MRI task. SCRs were sampled at $200 \mathrm{~Hz}$ using the Biopac (Goleta, CA) MP150/ GSR100C system, with Ag-AgCl electrode positioned on the distal (first) phalanges of the index and middle finger of the non-dominant hand and contact was facilitated with electrodermal GEL101.

The SCRs data were analyzed using AcqKnowledge 4.1.0 software for the MP150 system (Biopac system, Inc., Goletta, CA, USA). Data analysis procedures were conducted as described in the AcqKnowledge software manual. Anticipatory SCRs were measured, and the time window assessing the anticipatory SCRs was set to $5 \mathrm{~s}$ before a decision to allow SCRs to return to baseline, in accordance with Bechara et al. [32] and Katrin et al.[33]. Responses were measured in microSiemens $[\mu \mathrm{S}]$ and threshold was set to $0.1 \mu \mathrm{S}$ [34].

\section{5. fMRI data acquisition}

All MRI sessions were performed in the clinical MR imaging unit of René-Dubos Hospital center, Pontoise, France. Neuroimaging data were acquired with 1.5-Tesla, whole-body MRI system equipped with Signa head volume coil (MR450 magnet, General Electric Medical System, Milwaukee, WI). Functional images with BOLD contrast enhancement were acquired for each participant. Single-shot EPIs were acquired using a typical T2*-weighted gradient-echo sequence. Total of $2 \times 310$ EPI volumes $(\mathrm{TR} / \mathrm{TE}=3000 / 44 \mathrm{~ms}$; flip angle $=90$ degrees; matrix $=64 * 64 ; \mathrm{FOV}=240 \times 240 \mathrm{~mm}^{2}$ ) were obtained for both experimental runs performed at the beginning and at the end of the session, using forty four, 3.75-mm thick axial slices; isotropic voxel volume $=52.73 \mathrm{~mm}^{3}$. For structural images, conventional 3D imaging used an FSPGR BRAVO sequence (matrix: $256 \times 256$; flip angle: $12^{\circ}$; TR/TE: $8.6 / 3.3 \mathrm{~ms}$; FOV: $240 \times 240 \mathrm{~mm}^{2}$; 168 slices, $1 \mathrm{~mm}$ thick). We 
Side Effects
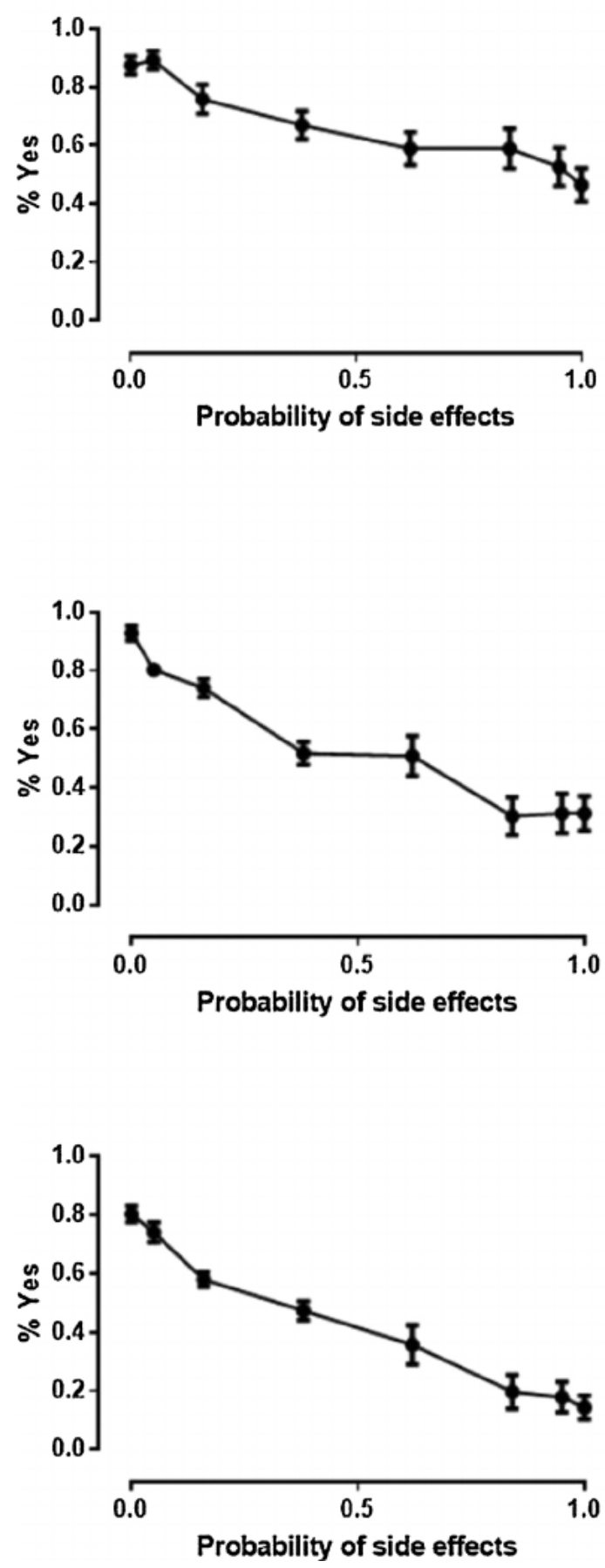

Efficacy
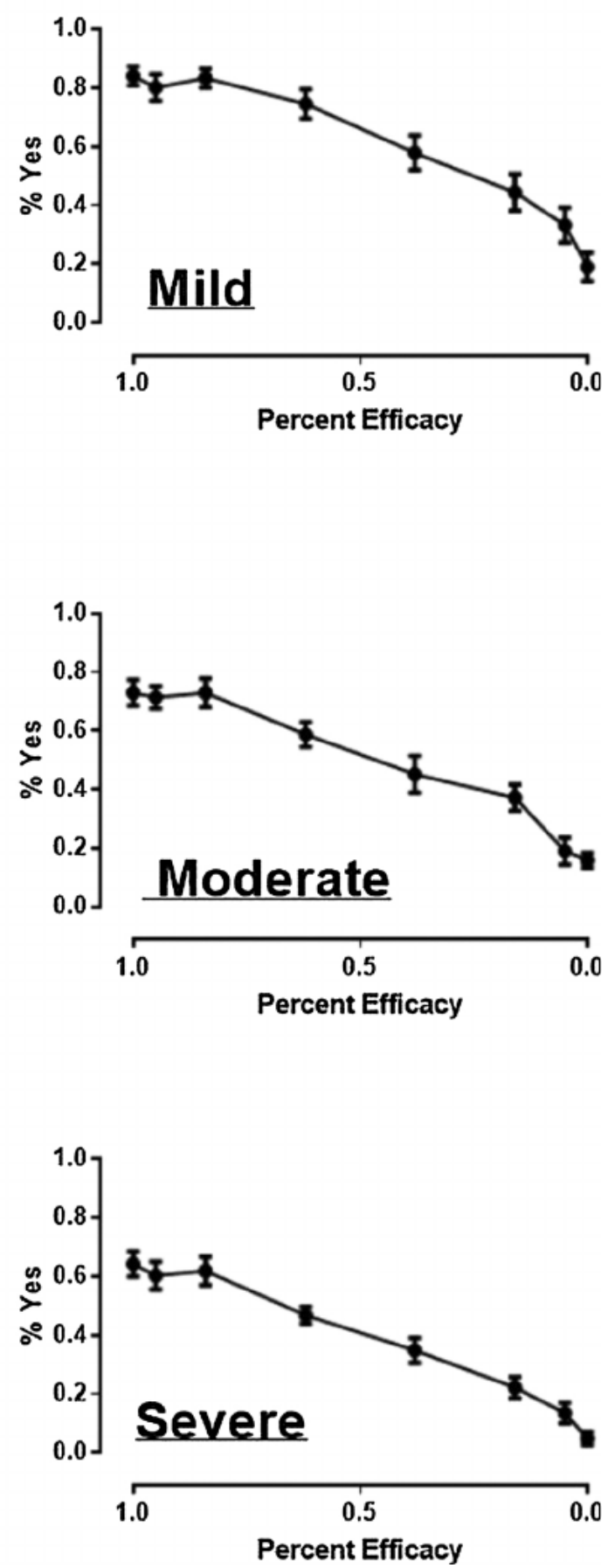

Fig. 2. The mean percent of "yes" responses at each side effect probability (left figures) and the percent "yes" responses at each percent efficacy (right figures) for questions involving mild (top graphs), moderate (middle graphs) and severe (bottom graphs) side effects. Error bars show the between subject SEM.

used a coronal-plane, perpendicular to the dominant field gradient, and a small voxel size to reduce the intra-voxel dephasing artifact due to the susceptibility-induced magnetic field in homogeneities, present in the vicinity of amygdala and orbitofrontal area [32].

\subsection{Image preprocessing and SPM analyses}

Image processing and data analysis were performed using Statistical Parametric Mapping SPM12 (http://www.fil.ion.ucl.ac.uk/spm/). For each participant, and for T1 equilibrium, the first four volume scans were discarded. All EPI volumes were corrected to adjust for withinvolume time differences and then realigned with the last volume to correct for head movements. Functional scans were then spatially normalized against the standard, stereotactic space of the MNI. Spatial smoothing was performed with an $8 \mathrm{~mm}$ full width half maximum Gaussian kernel. Hemodynamic responses were modeled as a canonical HRF convolved with a synthetic HRF.

A whole brain analysis was performed using a general linear model 


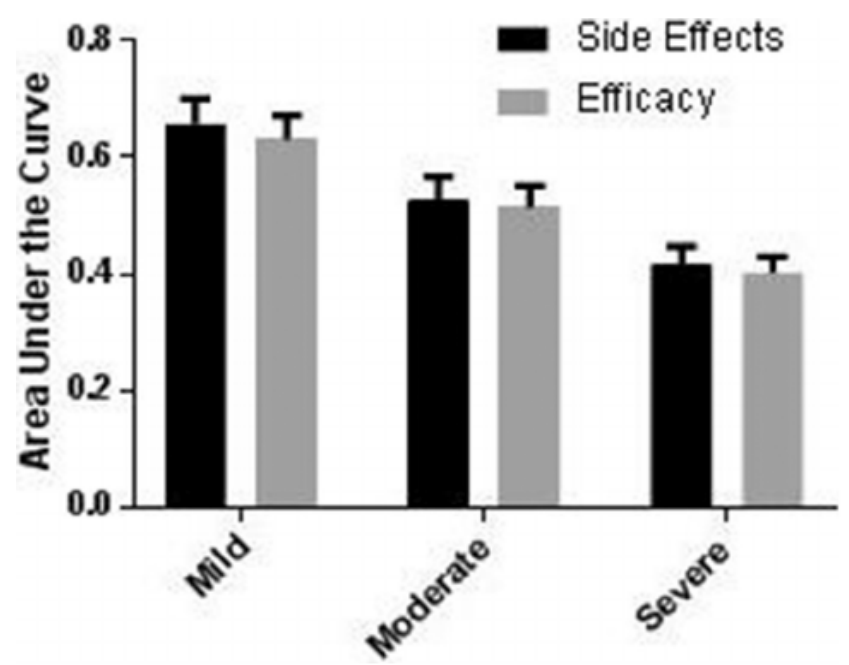

Fig. 3. Mean area under the curve (AUC) of side effects and efficacy probability at each side effects severities (mild, moderate and severe).

(GLM) approach [35]. For each participant, the motion parameters were included in the GLM as regressors and brain activation was examined for the contrast "mild compared to severe" and vice-versa. Regressors of interest were created by convolving a delta function representing the onset time of each scenario within each condition (mild and severe) with a canonical HRF. Onset time was defined as the trial onset for the stimulus presentation.

A single-subject, fixed-effect model was created for each individual participant, in order to perform the condition-based random-effects analysis. Results were declared significant at the peak level $(p<0.05$ corrected for multiple comparisons, family-wise error (FWE)).

Both brain activation changes and psychophysiological interaction (PPI) analysis were performed within the same dataset.

\subsection{Percent signal change and correlational analysis}

Percent signal change was measured on the mild and severe conditions for each subject and each region using the MarsBar toolbox (release 0.44). Extraction of the weighted mean signal was performed separately for each area. Correlational analysis of the percentage of BOLD signal change with AUC for each level of side effect severity (mild and severe) was performed for each participant within the VS, insula, amygdala and OFC as ascertained by the second-level random-effect analysis. Results were corrected for multiple comparisons using Bonferroni adjustment.

\subsection{Psychophysiological interaction analysis}

During a PPI analysis possible interactions between regression slopes of different brain areas can be significantly tested as a measure of effective connectivity [36].

The OFC served as the seed or hub region for all PPI analyses. This region was chosen because it has reciprocal anatomical connections with the VS, amygdala [16] and insula [37] and is implicated in reward anticipation as well as in risk processing [15,16,38].

Anatomical masks of the right OFC, bilateral insula, the right amygdala and the right VS were created using the Automated anatomical labeling (aal) atlas in the SPM Wake Forest University (WFU) PickAtlas toolbox (http://fmri.wfubmc.edu/software/ PickAtlas, version 2.5). The OFC mask included the superior, middle and medial parts of OFC, and the VS mask included the caudate and the putamen as defined in the aal atlas in the WFU PickAtlas toolbox.

Extracted haemodynamic time series from seed regions were deconvolved and the resulting neuronal time series (physiological variable) were combined with the cue onset times for each stimulus presented under the mild side effect and severe side effect stimulus condition (psychological variables) to derive the interaction term (source signal * experimental context). To test for differences in regression slopes between the two experimental conditions, a GLM was generated with this interaction term as the explanatory variable (SPM12 Manuel, chapter36, http://www.fil.ion.ucl.ac.uk/spm/). The resulting individual t-contrast images were entered into a random effects group analysis and tested for statistical significance at $p<0.05$ (FWE-corrected).

\section{Results}

\subsection{Behavioral data}

Fig. 2 shows the proportion of "yes" responses at each side effect probability (left column) and at each level of efficacy (right column) when the side effects were mild (top row) moderate (middle row) and severe (bottom row). As can be seen, the proportion of "yes" responses decreased as a function of both increasing side effect probability and decreasing efficacy. Moreover, the overall percent of yes responses decreased as side effects became more severe. When summarized as AUC (Fig. 3), these side effect severity-based decrements in willingness to take medications did not differ by side effect vs. efficacy AUCs.

A repeated measured ANOVA showed a significant main effect of side effect severity $(<0.0001)$, but not side effect vs efficacy $(p=.73)$ nor an interaction $(p=.91)$.

\subsection{SCRs results}

SCRs values were significantly higher when people made medication decisions in the severe side effects condition compared with mild side effects condition $(p<0.01)$.

Significant negative correlations between anticipatory SCRs (Mean $=1.42, \quad \mathrm{SD}=0.62$ ) and $\mathrm{AUC}$ side effects (Mean $=0.64$; $\mathrm{SD}=0.16 ; \mathrm{r}=-0.84$ at $p<0.05)$ and between anticipatory SCRs /AUC efficacy (Mean $=0.62 ; \mathrm{SD}=0.16 ; \mathrm{r}=-0.69$ at $p<0.05$ ) were recorded in the severe condition. Surprisingly, significant negative correlations between anticipatory SCRs (Mean $=0.49$; $\mathrm{SD}=0.26$ ) and AUCs side effects $(\mathrm{r}=-0.69$ at $p<0.05)$, and between anticipatory SCRs/AUCs efficacy $(r=-0.67$ at $p<0.05$ ) were also observed in the mild condition.

\subsection{Brain activation}

When compared to the severe side effects condition, participants making decisions in the mild side effects condition had increased activation within the OFC (BA11) and the VS at $p<0.05$ (FWE-corrected) (Table1, Fig. 4). When compared to the mild side effect condition, participants making decisions in the severe condition had increased

Table 1

Results of main activated regions for the contrasts "mild > severe" and "severe > mild".

\begin{tabular}{|c|c|c|c|c|c|c|c|}
\hline \multirow[t]{2}{*}{ Regions } & \multirow[t]{2}{*}{ Side } & \multirow[t]{2}{*}{ BA } & \multicolumn{3}{|c|}{ MNI Coordinates } & \multirow[t]{2}{*}{$\mathrm{t}$} & \multirow[t]{2}{*}{$\mathrm{P}(\mathrm{FWE})$} \\
\hline & & & $\mathrm{x}$ & $\mathrm{y}$ & $\mathrm{z}$ & & \\
\hline \multicolumn{8}{|c|}{ Mild > Severe contrast } \\
\hline VS & Right & $*$ & 21 & 14 & -5 & 3.57 & 0.024 \\
\hline OFC & Right & 11 & 24 & 44 & -11 & 3.25 & 0.040 \\
\hline \multicolumn{8}{|c|}{ Severe $>$ Mild contrast } \\
\hline \multirow{2}{*}{ Insula } & Right & 13 & 42 & -34 & 19 & 4.25 & 0.006 \\
\hline & Left & 13 & -45 & 8 & -5 & 3.17 & 0.042 \\
\hline Amygdala & Right & $*$ & 27 & -4 & -11 & 3.23 & 0.038 \\
\hline Thalamus & Right & $*$ & 12 & -10 & 4 & 3.42 & 0.027 \\
\hline OFC & Right & 47 & 21 & 26 & -2 & 3.13 & 0.044 \\
\hline
\end{tabular}




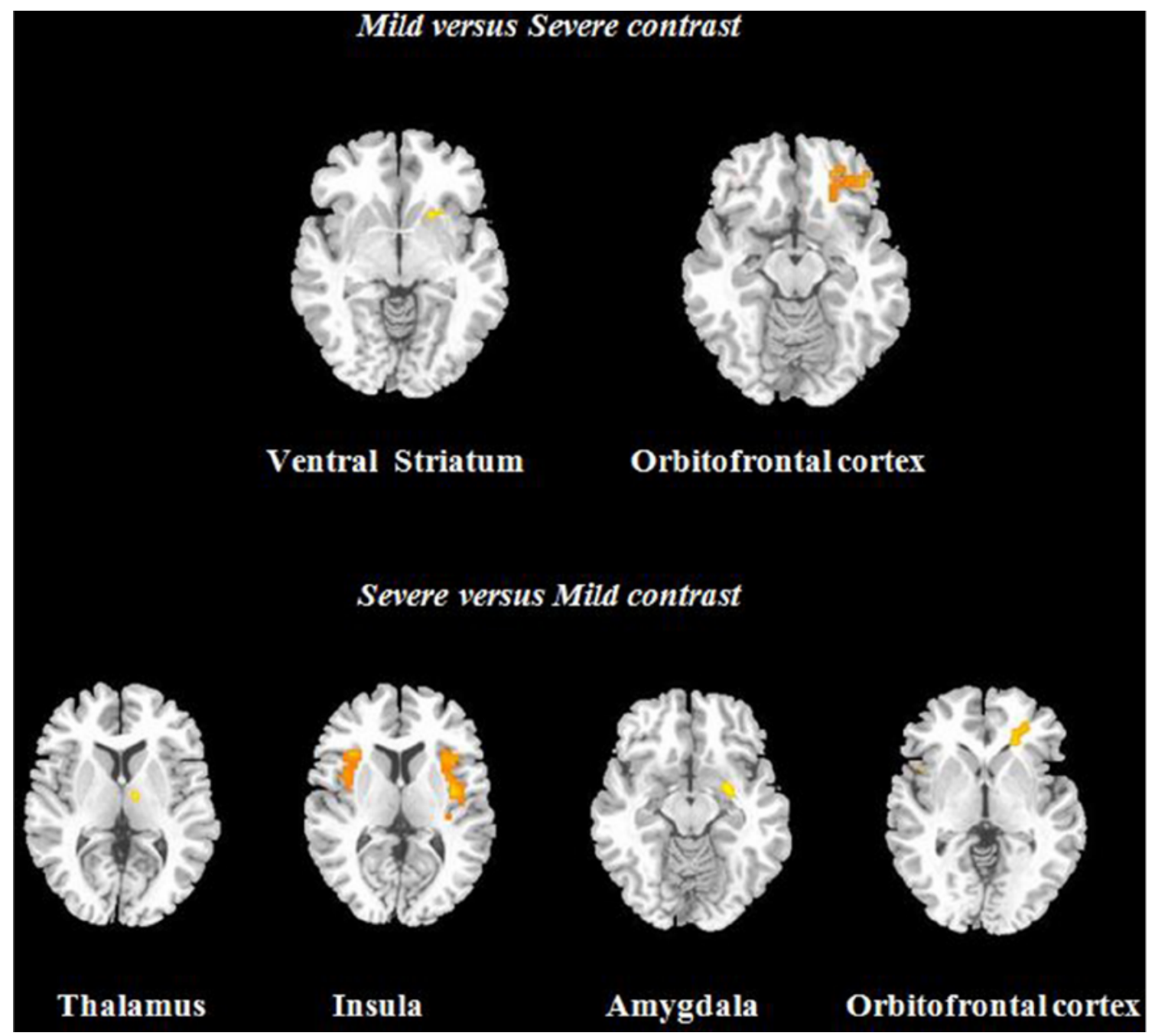

Fig. 4. T1-weighted MR images shows activations in the OFC and the VS in the "mild vs severe" contrast, and activation in the OFC, amygdala, insula and thalamus in the "severe vs mild" contrast at $p<0.05$ (FWE-corrected). The coordinates of the maximum activated voxels are listed in Table 1.

activation in the OFC (BA47), amygdala, insula and thalamus at $p<0.05$ (FWE-corrected) (Table 1, Fig. 4).

\subsection{Correlational results of BOLD signal change with AUC side effects /} efficacy

Fig. 5 shows the relation between AUCs (y-axis; based on side effect probability (open circles) and efficacy (closed circles) and percent signal change (x-axis) in OFC (top row), VS (second row), insula (third row) and Amygdala (bottom row) when side effects were mild (left column) versus severe (right column) (Pearson's r; Table2).

Significant positive correlations between AUCs and percent signal change in the OFC and VS were observed, particularly when the side effects were mild. These correlations became less pronounced in the severe side effect condition. By contrast correlations between percent signal change in insula and amygdala and AUCs were generally negative, and became more pronounced in the severe side effect condition (Fig. 5).

\subsection{PPI results}

During processing of the mild side effect condition, PPI results showed increased effective connectivity between the OFC and VS as compared to the severe side effect condition. The peak voxel of the VS was found at $\mathrm{x}=14, \mathrm{y}=23, \mathrm{z}=-2, p<0.05$ (FWE-corrected).
These voxels were tested and found to be more active during the mild vs severe condition (Fig. 6).

However, PPI analysis showed increased effective connectivity between the OFC and amygdala and between the OFC and insula when subjects engaged in the severe side effect condition. The peak voxel of the amygdala was found at $\mathrm{x}=21, \mathrm{y}=-2, \mathrm{z}=-15, p<0.05$ (FWEcorrected) (Fig. 6). The peak voxel of the insula was found at $x=44$, $\mathrm{y}=-2, \mathrm{z}=8, p<0.05$ (FWE-corrected). These voxels were found to be more active during PPI analyses of the severe versus mild side effect condition (Fig. 6).

\section{Discussion}

The aim of this study was to examine the neural underpinnings of how healthy adults weigh risks (side effects) and benefits (efficacy) when making treatment choices.

Our results confirm a role of OFC and VS in health decision making. Functionally, VS has repeatedly been implicated in processing and encoding the value of a chosen action $[15,39,40]$. Studies have shown that striatal neurons have reward-specific activity and also process temporal and probabilistic information regarding the occurrence of reward signals. In contrast, recent studies have implicated VS in coding prediction error rather than reflecting the subjective values of competing choices [41]. For instance, reward prediction error signaling is frequently witnessed in VS during human fMRI studies of learning and decision- 


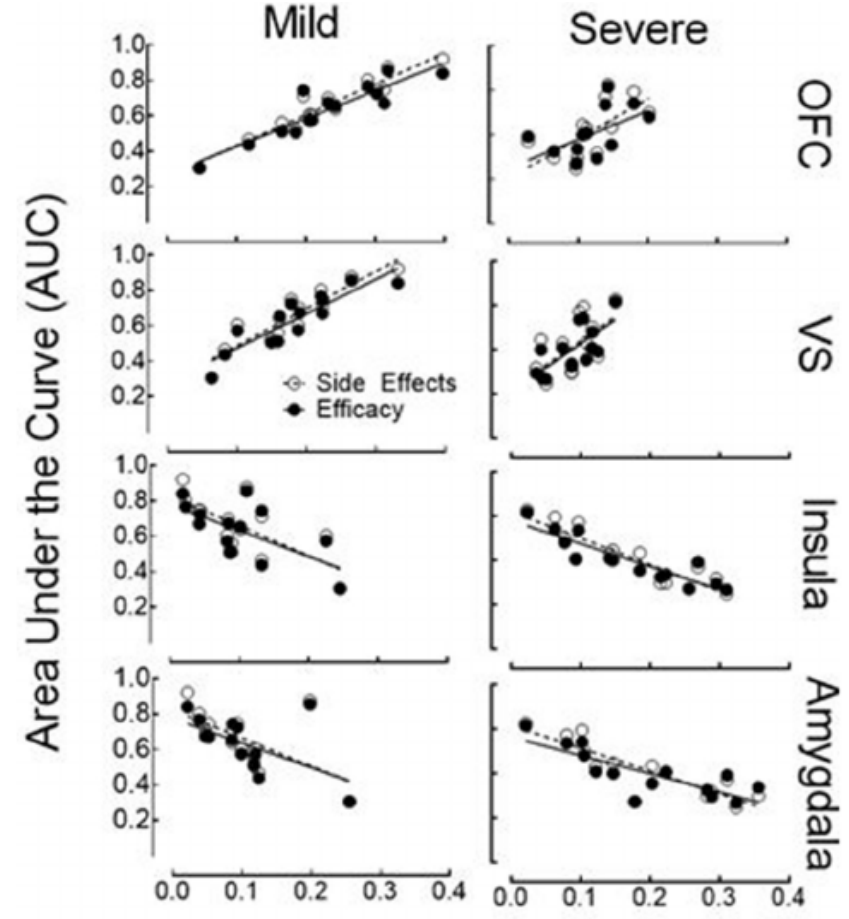

\section{Percent Signal Change}

Fig. 5. Correlational analysis between the AUC values for side effects (open circles, dashed lines) and efficacy (closed circles, solid line) with percent of BOLD signal change, with AUCs for the mild side effects being in the left column, and severe side effects being in the right column and percent signal change in the OFC in the top row, VS the second row, insula third row, and amygdala being the bottom row.

Table 2

Correlation coefficients (pearson's r) between BOLD signal change and AUC side effects / AUC efficacy. Statistical significance at $p<0.01$.

\begin{tabular}{lllllr}
\hline & \multicolumn{2}{l}{ Mild } & & \multicolumn{2}{l}{ Severe } \\
\cline { 2 - 3 } \cline { 5 - 6 } & Side Effects & Efficacy & & Side Effects & Efficacy \\
\hline OFC & 0.954 & 0.908 & & 0.739 & 0.665 \\
VS & 0.916 & 0.891 & & 0.746 & 0.669 \\
Insula & -0.730 & -0.670 & & -0.899 & -0.901 \\
Amygdala & -708 & -0.663 & & -0.866 & -0.087 \\
\hline
\end{tabular}

making, and has been found to act as a teaching signal that updates expected reward values, therefore driving behavior and decision making [42]. OFC is also known to integrate information from different sensory modalities [39] and estimate the reward value of ongoing events $[16,43]$. While, the subjective value of reward and prediction error are often highly correlated, both regions (OFC and VS) are thought to be implicated in coding prediction error [44]. However, studies have highlited the dissociated role of both region, with OFC involved in the valuation and processing of reward outcomes [45,46], whereas, the VS is involved in anticipation and reward-related predition error $[41,46,47]$. In line with these findings, many studies highlight the fact that the reward network functions as an interplay between reward anticipation processing in VS and reward outcome processing in OFC $[47,48]$. Our findings are consistent with this understanding of VS and OFC playing a primary role in reward salience.

Together, OFC and VS are involved in reward anticipation/processing and form a domain general valuation network $[15,16]$. Both are considered central nodes in this reward network because they are the primary projection areas of the meso-limbic and meso-cortical dopaminergic pathways [23,49].

Recent fMRI studies demonstrate that OFC and VS exhibit strong functional connectivity [49,50], and clinical research shows an increased effective connectivity between OFC and VS (NAcc) among obese women when presenting food images associated with reward processing. This connectivity is strengthened by increased dopamine function within VS which acts to gate reward inputs from the OFC to VS. The high level of activity within OFC become more effective in further increasing VS activity [51]. Our PPI results confirm that this network also plays a role in medical decision making when people weigh potential side effects and medication efficacies. Specifically, OFC and VS demonstrated a strong effective connectivity in relative reward (mild side effects) compared to relative risk (severe side effects) conditions, and were associated with increased likelihood of choosing to take medication (higher AUC side effects and efficacy).

By contrast, in the severe side effect condition, our results showed significant activation within the fronto-limbic system including OFC, amygdala, insula and the thalamus. The activation of this circuit was associated with decreased likelihood of choosing to take medication (lower AUC), and might reflect the emotional anticipation of suffering worse quality of life caused by adverse medication side effects. Previous studies are in accordance with our findings, for instance showing OFC activation in response to both potentially rewarding and aversive stimuli [52]. Some OFC neurons respond more strongly to rewards and others to punishment, reflecting a convergence of appetitive and aversive information in the brain [52]. Similarly, the thalamus is highly implicated in the processing of aversive emotion such as regret [53] and was associated with risk processing and risk representation [1].

The insula and the amygdala have repeatedly been implicated in the anticipation of punishment [54], the anticipation of negative outcomes [55], and aversive events [56]. More importantly, recent studies have confirmed the crucial role of the insula and amygdala in guiding appropriate choices [57].

Preclinical and clinical evidence shows that the amygdala is involved in risk-based decision-making $[38,58]$ and patients with amygdala damage make more risky choices than controls in laboratory gambling tasks [3,32].

Similarly, several studies have supported the involvement of bilateral insula in decision making [2], risk and risk prediction error [59], and it has been shown that posterior insula is a critical component of the decision-making neural network and is involved in delay discounting tasks [60]. Moreover, activation within the posterior insula was increased while presenting negative images and was correlated with greater negative affect ratings [61].

Anatomically, insula and amygdala are well positioned to play an integrative role in linking affective value with adaptive behavior, because they possesses bidirectional connections with numerous structures implicated in reward and decision making, including OFC [62]. The insula forms an integrated representation of bodily states [63] and changes of insular activity could modulate decision-making [19]. Further, neural networks including the insular cortex might involve modulation of exploration in decision-making on the basis of coding and evaluation of uncertainty [64]. Consistent with these findings, the anticipation of negative bodily states triggers interoceptive signals in the insula $[65,66]$ and these signals are transmitted to OFC where information is maintained on-line to inform decisions and guide actions [67]. Similarly, sharing strong reciprocal connections with OFC $[51,68]$, the amygdala and OFC probably provide complimentary information on the reward value of stimuli and events [69]. The amygdala is thought to detect and recognize the valence of affective stimuli and then feed forward this information to OFC. Our PPI findings suggest that similar processes occur when people weigh the risks associated with medical treatments. The positive connectivity between OFC-insula and OFC-amygdala demonstrates that both regions exhibit co-activation during the severe side effect condition when compared to the mild side effect condition, and these neural interactions were stronger among 
A)
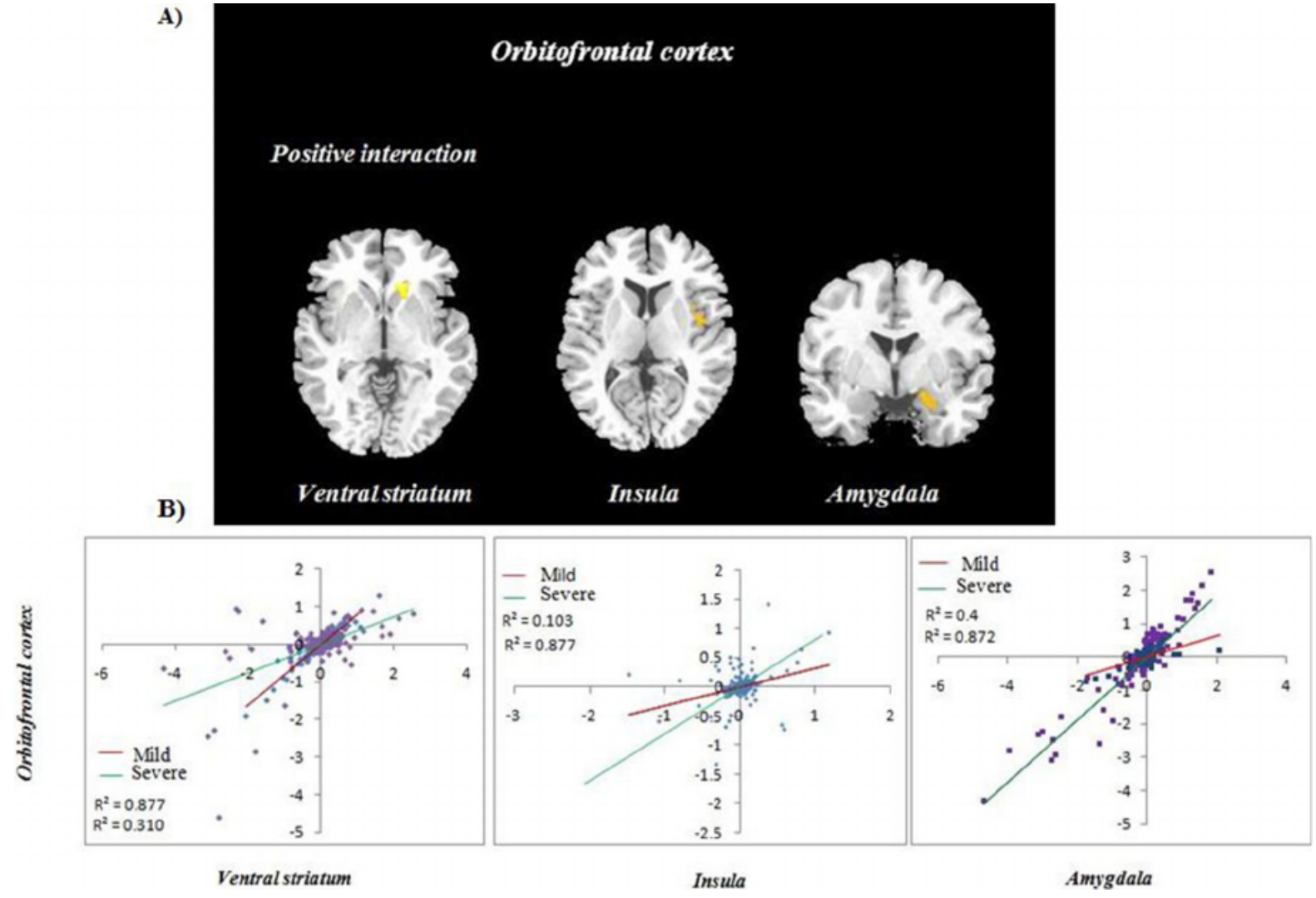

Fig. 6. Results of the psycho-physiological interaction (PPI) analysis between OFC-VS in "mild vs severe" condition and between OFC-amygdala, OFC-insula in "severe vs mild" condition. (A) T1-weighted MR images shows activation within the VS, the amygdala and the insula at p < 0.05 (FWE-corrected). (B) Slopes found between the OFC-VS in "mild vs severe" condition, and between the OFC-amygdala and the OFC- insula in "severe vs mild" condition are significantly different $(\mathrm{p}<0.05)$ (FWE-corrected).

people who quickly decided to not take medicines as side effect probabilities increased.

Our behavioral results are consistent with our previous work using probability discounting to model treatment decisions among patients with MS [8,9,22], and demonstrate that participants are more likely to refuse medication when side effects are high and efficacy is low. Our results confirm the idea that participants discount the subjective value of reward as the likelihood of its receipt decreases [70]. More importantly, individual differences in discounting rates were significantly correlated with connectivity in the postulated risk and reward neural networks.

The affective results support the behavioral and fMRI data, and show that participants generate increased anticipatory SCRs before making riskier decisions (in our case; when medication side effects were severe). Our results are in accordance with previous studies which have shown that healthy subjects tend to generate heightened SCRs before they choose from risky decks [18], and are in line with Damasio's theory that emphasizes the importance of emotions in guiding decisionmaking processes.

This study represents a first step in understanding the neural underpinnings of how people weigh risks and benefits when making medical decisions. As such, there are several limitations and areas for future research. First, despite reaching statistical significance with correction for family wise error, future studies should include larger, more diverse samples to confirm these preliminary findings. Second, the current investigation was a pilot study that asked healthy adult participants to make hypothetical treatment decisions. Several lines of research indicate that hypothetical/imagined scenarios activate the same brain networks as more consequential scenarios that have real world implications [25-28]. Consistent with this research, despite using a hypothetical scenario, our results activated brain networks known to be associated with consumer and risk-based decision-making [1-4]. While this study uncovered brain networks associated with how people weigh treatment risks and benefits, replication in a clinical sample is recommended. We suspect that applying this paradigm to patients may elicit even stronger findings. For instance, patients with prior treatment experience may react more strongly to treatment choices that include the possibility of severe side effects, possibly showing increased connectivity between OFC and insula/amygdala.

\section{Conclusion}

Our results demonstrate the role of orbitofrontal cortex and ventral striatum when adult participants ascribe increased value to treatment, and implicate OFC, amygdala and insula in treatment-related risk anticipation.

This study represents a first step in understanding the neural underpinnings of how patients weigh risks and benefits when making treatment decisions. Future studies should confirm these results with a larger sample of patients with chronic disease. Ultimately, an improved understanding of the neural bases for treatment decision-making may lead to clinical advances that improve adherence among patients with chronic disease.

\section{Conflict of interest}

The authors of this manuscript declare that they have no conflict of interest to disclose. 


\section{Acknowledgements}

This work was partly supported by the COMUE UNIVERSITÉ PARIS LUMIÈRES(UPL). Dr. Bruce provides unbranded talks for Novartis and has received funding from the National Multiple Sclerosis Society.

\section{References}

[1] P.N. Mohr, G. Biele, H.R. Heekeren, Neural processing of risk, J. Neurosci. 30 (19) (2010) 6613-6619.

[2] P.N. Mohr, et al., Neural foundations of risk-return trade-off in investment decisions, Neuroimage 49 (3) (2010) 2556-2563.

[3] M. Hsu, et al., Neural systems responding to degrees of uncertainty in human decision-making, Science 310 (5754) (2005) 1680-1683.

[4] J. Peters, C. Buchel, Overlapping and distinct neural systems code for subjective value during intertemporal and risky decision making, J. Neurosci. 29 (50) (2009) $15727-15734$.

[5] B. Gardner, P. Lally, J. Wardle, Making health habitual: the psychology of 'habitformation' and general practice, Br. J. Gen. Pract. 62 (605) (2012) 664-666.

[6] J.M. Bruce, S.G. Lynch, Multiple sclerosis: MS treatment adherence-how to keep patients on medication? Nat. Rev. Neurol 7 (8) (2011) 421-422.

[7] J. Wong, et al., Adherence to multiple sclerosis disease-modifying therapies in Ontario is low, Can. J. Neurol. Sci. 38 (3) (2011) 429-433.

[8] J.M. Bruce, et al., Being kind to your future self: probability discounting of health decision-making, Ann. Behav. Med. 50 (2) (2016) 297-309.

[9] D.P. Jarmolowicz, et al., Using EP50 to forecast treatment adherence in individuals with multiple sclerosis, Behav. Processes 132 (2016) 94-99.

[10] K.L. Hendrickson, E.B. Rasmussen, Effects of mindful eating training on delay and probability discounting for food and money in obese and healthy-weight individuals, Behav. Res. Ther. 51 (7) (2013) 399-409.

[11] H. Rachlin, A. Raineri, D. Cross, Subjective probability and delay, J. Exp. Anal. Behav. 55 (2) (1991) 233-244.

[12] D.V. Poltavski, J.N. Weatherly, Delay and probability discounting of multiple commodities in smokers and never-smokers using multiple-choice tasks, Behav. Pharmacol. 24 (8) (2013) 659-667.

[13] M.W. Johnson, et al., Delay and probability discounting of sexual and monetary outcomes in individuals with cocaine use disorders and matched controls, PLoS One 10 (5) (2015) e0128641.

[14] S. De Geest, E. Sabate, Adherence to long-term therapies: evidence for action, Eur. J. Cardiovasc. Nurs. 2 (4) (2003) 323.

[15] J.W. Kable, P.W. Glimcher, The neurobiology of decision: consensus and controversy, Neuron 63 (6) (2009) 733-745.

[16] M.L. Kringelbach, E.T. Rolls, The functional neuroanatomy of the human orbitofrontal cortex: evidence from neuroimaging and neuropsychology, Prog. Neurobiol. 72 (5) (2004) 341-372.

[17] A. Bechara, H. Damasio, A.R. Damasio, Emotion, decision making and the orbitofrontal cortex, Cereb. Cortex 10 (3) (2000) 295-307.

[18] A.R. Damasio, The somatic marker hypothesis and the possible functions of the prefrontal cortex, Philos. Trans. R. Soc. Lond. B Biol. Sci. 351 (1346) (1996) 1413-1420.

[19] A.R. Damasio, Descartes' Error : Emotion, Reason, and the Human Brain, Putnam, New York, 1994, p. 312 xix.

[20] S.M. McClure, et al., Separate neural systems value immediate and delayed monetary rewards, Science 306 (5695) (2004) 503-507.

[21] S.M. McClure, et al., Time discounting for primary rewards, J. Neurosci. 27 (21) (2007) 5796-5804.

[22] D.P. Jarmolowicz, et al., On how patients with multiple sclerosis weigh side effect severity and treatment efficacy when making treatment decisions, Exp. Clin. Psychopharmacol. 25 (6) (2017) 479-484.

[23] F. Cauda, et al., Functional connectivity and coactivation of the nucleus accumbens: a combined functional connectivity and structure-based meta-analysis, J. Cogn. Neurosci. 23 (10) (2011) 2864-2877.

[24] P.L. Gabbott, et al., Prefrontal cortex in the rat: projections to subcortical autonomic, motor, and limbic centers, J. Comp. Neurol 492 (2) (2005) 145-177.

[25] M.L. Locey, B.A. Jones, H. Rachlin, Real and hypothetical rewards, Judgm. Decis. Mak. 6 (6) (2011) 552-564.

[26] K.P. Miyapuram, et al., BOLD responses in reward regions to hypothetical and imaginary monetary rewards, Neuroimage 59 (2) (2012) 1692-1699.

[27] A.K. Matusiewicz, et al., Statistical equivalence and test-retest reliability of delay and probability discounting using real and hypothetical rewards, Behav. Processes 100 (2013) $116-122$

[28] C.H. Bowman, O.H. Turnbull, Real versus facsimile reinforcers on the Iowa gambling task, Brain Cogn. 53 (2) (2003) 207-210.

[29] L. Green, J. Myerson, A discounting framework for choice with delayed and probabilistic rewards, Psychol. Bull. 130 (5) (2004) 769-792.

[30] Y. Ohmura, T. Takahashi, N. Kitamura, Discounting delayed and probabilistic monetary gains and losses by smokers of cigarettes, Psychopharmacology (Berl) 182 (4) (2005) 508-515.

[31] J. Myerson, L. Green, M. Warusawitharana, Area under the curve as a measure of discounting, J. Exp. Anal. Behav. 76 (2) (2001) 235-243.

[32] A. Bechara, et al., Different contributions of the human amygdala and ventromedial prefrontal cortex to decision-making, J. Neurosci. 19 (13) (1999) 5473-5481.

[33] K. Starcke, et al., Skin conductance responses during decisions in ambiguous and risky situations in obsessive-compulsive disorder, Cogn. Neuropsychiatry 14 (3) (2009) 199-216.
[34] P. Trotzke, et al., Impaired decision making under ambiguity but not under risk in individuals with pathological buying-behavioral and psychophysiological evidence, Psychiatry Res. 229 (1-2) (2015) 551-558.

[35] C.R. Pernet, Misconceptions in the use of the general linear model applied to functional MRI: a tutorial for junior neuro-imagers, Front. Neurosci. (8) (2014) 1.

[36] K.J. Friston, et al., Psychophysiological and modulatory interactions in neuroimaging, Neuroimage 6 (3) (1997) 218-229.

[37] M.M. Mesulam, E.J. Mufson, Insula of the old world monkey. III: efferent cortical output and comments on function, J. Comp. Neurol. 212 (1) (1982) 38-52.

[38] S.B. Floresco, et al., Cortico-limbic-striatal circuits subserving different forms of cost-benefit decision making, Cogn. Affect Behav. Neurosci. 8 (4) (2008) 375-389.

[39] J.P. O'Doherty, et al., Predictive neural coding of reward preference involves dissociable responses in human ventral midbrain and ventral striatum, Neuron 49 (1) (2006) 157-166.

[40] J.W. Kable, P.W. Glimcher, The neural correlates of subjective value during intertemporal choice, Nat. Neurosci. 10 (12) (2007) 1625-1633.

[41] T.A. Hare, et al., Dissociating the role of the orbitofrontal cortex and the striatum in the computation of goal values and prediction errors, J. Neurosci. 28 (22) (2008) 5623-5630.

[42] E.C. Brown, M. Brune, The role of prediction in social neuroscience, Front. Hum. Neurosci. (6) (2012) 147.

[43] H. Walter, et al., Motivating forces of human actions. Neuroimaging reward and social interaction, Brain Res. Bull. 67 (5) (2005) 368-381.

[44] J.P. O'Doherty, et al., Temporal difference models and reward-related learning in the human brain, Neuron 38 (2) (2003) 329-337.

[45] M.L. Kringelbach, et al., Activation of the human orbitofrontal cortex to a liquid food stimulus is correlated with its subjective pleasantness, Cereb. Cortex 13 (10) (2003) 1064-1071.

[46] B. Knutson, et al., Dissociation of reward anticipation and outcome with eventrelated fMRI, Neuroreport 12 (17) (2001) 3683-3687.

[47] W. Schultz, P. Dayan, P.R. Montague, A neural substrate of prediction and reward, Science 275 (5306) (1997) 1593-1599.

[48] A. Lee, R.M. Gilbert, Epidemiology of Parkinson disease, Neurol. Clin. 34 (4) (2016) 955-965.

[49] E.Y. Choi, B.T. Yeo, R.L. Buckner, The organization of the human striatum estimated by intrinsic functional connectivity, J. Neurophysiol. 108 (8) (2012) $2242-2263$.

[50] M.S. Pujara, et al., Ventromedial prefrontal cortex damage is associated with decreased ventral striatum volume and response to reward, J. Neurosci. 36 (18) (2016) 5047-5054.

[51] L.E. Stoeckel, et al., Effective connectivity of a reward network in obese women, Brain Res. Bull 79 (6) (2009) 388-395.

[52] S.E. Morrison, C.D. Salzman, The convergence of information about rewarding and aversive stimuli in single neurons, J. Neurosci. 29 (37) (2009) 11471-11483.

[53] P.V. Chandrasekhar, et al., Neurobiological regret and rejoice functions for aversive outcomes, Neuroimage 39 (3) (2008) 1472-1484.

[54] B. Seymour, T. Singer, R. Dolan, The neurobiology of punishment, Nat. Rev. Neurosci. 8 (4) (2007) 300-311.

[55] T.D. Wager, et al., Placebo-induced changes in FMRI in the anticipation and experience of pain, Science 303 (5661) (2004) 1162-1167.

[56] J.B. Nitschke, et al., Functional neuroanatomy of aversion and its anticipation, Neuroimage 29 (1) (2006) 106-116.

[57] H.C. Schiff, et al., An insula-central amygdala circuit for guiding tastant-reinforced choice behavior, J. Neurosci. 38 (6) (2018) 1418-1429.

[58] J.L. Gowin, S. Mackey, M.P. Paulus, Altered risk-related processing in substance users: imbalance of pain and gain, Drug Alcohol. Depend. 132 (1-2) (2013) 13-21.

[59] K. Preuschoff, S.R. Quartz, P. Bossaerts, Human insula activation reflects risk prediction errors as well as risk, J. Neurosci. 28 (11) (2008) 2745-2752.

[60] M. Wittmann, D.S. Leland, M.P. Paulus, Time and decision making: differential contribution of the posterior insular cortex and the striatum during a delay discounting task, Exp. Brain Res. 179 (4) (2007) 643-653.

[61] B.T. Denny, et al., Insula-amygdala functional connectivity is correlated with habituation to repeated negative images, Soc. Cogn. Affect Neurosci. 9 (11) (2014) $1660-1667$.

[62] S.M. Reynolds, D.S. Zahm, Specificity in the projections of prefrontal and insular cortex to ventral striatopallidum and the extended amygdala, J. Neurosci. 25 (50) (2005) 11757-11767.

[63] A.D. Craig, How do you feel-now? The anterior insula and human awareness, Nat. Rev. Neurosci. 10 (1) (2009) 59-70

[64] H. Ohira, et al., Neural and sympathetic activity associated with exploration in decision-making: further evidence for involvement of insula, Front. Behav. Neurosci. (8) (2014) 381.

[65] N.H. Naqvi, et al., Damage to the insula disrupts addiction to cigarette smoking, Science 315 (5811) (2007) 531-534.

[66] H.D. Critchley, et al., Neural systems supporting interoceptive awareness, Nat. Neurosci. 7 (2) (2004) 189-195.

[67] J. Tanabe, et al., Insula and orbitofrontal cortical morphology in substance de pendence is modulated by sex, AJNR Am. J. Neuroradiol. 34 (6) (2013) 1150-1156.

[68] A.L. Gold, R.A. Morey, G. McCarthy, Amygdala-prefrontal cortex functional connectivity during threat-induced anxiety and goal distraction, Biol. Psychiatry 77 (4) (2015) 394-403.

[69] M.G. Baxter, et al., Control of response selection by reinforcer value requires interaction of amygdala and orbital prefrontal cortex, J. Neurosci. 20 (11) (2000) 4311-4319.

[70] S. Jones, M. Oaksford, Preferences show greater stability for transactions than for gambles in cost discounting, Front. Psychol. (2) (2011) 293. 